\title{
Exercise as Medicine in Multiple Sclerosis- Moving Beyond Compensatory Benefits
}

\author{
Kathleen M Zackowski, PhD, OTR, MSCS \\ Senior Director, Patient Management, Care and Rehabilitation Research, National Multiple Sclerosis Society, New York, NY, US
}

DOI: https://doi.org/10.17925/USN.2017.13.02.70

$\mathrm{M}$ any studies have shown that physical exercise improves multiple sclerosis (MS) symptoms, including cognition, fatigue, bowel and bladder function, depression, and overall quality of life. However, the pathophysiologic mechanisms responsible for these symptomatic changes remain elusive. Exercise offers a likely path for addressing progressive MS, nervous system repair, and as a wellness approach for people with MS. We as a community need to work toward gaining information to establish an evidence-based exercise prescription for people living with MS.

\section{Keywords}

Physical activity, training, aerobic exercise, strength training, plasticity, rehabilitation, recovery

Disclosure: Kathleen M Zackowski has nothing to declare in relation to this article. This article is a short opinion piece and has not been submitted to external peer reviewers. No funding was received in the publication of this article.

Authorship: All named authors meet the International Committee of Medical Journal Editors (ICMJE) criteria for authorship of this manuscript, take responsibility for the integrity of the work as a whole, and have given final approval for the version to be published.

Open Access: This article is published under the Creative commons Attribution Noncommercial License, which permits any noncommercial use, distribution, adaptation, and reproduction provided the original author(s) and source are given appropriate credit.

Received: May 31, 2017

Published Online: July 24, 2017

Citation: US Neurology, 2017;13(2):70-1

Corresponding Author: Kathleen M Zackowski, Patient Management, Care and Rehabilitation Research, National Multiple Sclerosis Society, 733 3rd Avenue, 3rd Floor, New York, NY 10017, US. E: kathleen.zackowski@nmss.org
The importance of exercise for symptom management in multiple sclerosis (MS) has gained increasing momentum over the last 25 years. It was not so long ago that patients with MS were advised to avoid exercise. Studies in the 1970s showed evidence suggesting that increasing body temperature when exercising caused variable changes in motor function for people with MS. ${ }^{1}$ However, in 1996, Petajan and colleagues ${ }^{2}$ highlighted the benefits of exercise, showing evidence that aerobic training improved cardiovascular fitness, bowel and bladder function, fatigue, depression, and increased participation in social activities. Since then many studies have described symptomatic benefits gained when using different exercise modalities..$^{3-7}$ Frohman and colleagues ${ }^{8}$ described how aquatic exercise provides a unique and beneficial medium for patients with MS due to water's buoyant effects and the thermodynamic, or heat dissipating, benefits associated with exercising in cool water.

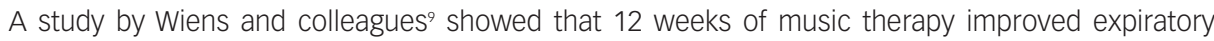
muscle strength by training participants with MS to breathe diaphragmatically and coordinate breath with speech. One study showed that benefits from exercise could be gained in as little as 8 weeks with supervised strength training. ${ }^{10}$ It is important to emphasize that MS symptoms are often improved when people exercise.

Given these positive changes in MS symptoms it is unfortunate that our understanding of the pathophysiologic pathways that can explain the positive effects of exercise remains unclear Exercise is used often in MS clinical care; however, the focus is on its compensatory benefits without regard to its potential restorative benefits. We need answers to questions such as: Does physical exercise improve MS symptoms via synaptic changes at the axon? Does exercise effect cortical reorganization in MS? Are metabolic changes the important element, or does exercise simply improve co-morbidities (e.g., weight loss), thereby indirectly affecting secondary complications such as pain? Evaluating the link between symptoms and brain and spinal cord pathology in people with MS has been done almost exclusively using imaging technology. There is evidence that specific areas of the brain and spinal cord correlate with behavioral outcome measures such as strength, fatigue, and cognition..$^{11-13}$ Yet it has proved difficult to move past descriptions of correlational relationships. Rodent experimental autoimmune encephalomyelitis models offer another avenue to evaluate mechanism and behavior. One study in mice showed that voluntary wheel running (i.e., aerobic exercise) significantly attenuated the magnitude of axonal loss when compared to a nonrunning condition, ${ }^{14}$ and showed an overall reduction in oxidative stress. ${ }^{15,16}$ Evidence from other pathologies should also be considered, and if relevant, pursued in MS. Jakowec and colleagues ${ }^{17}$ showed that motor recovery in patients with Parkinson's disease is enhanced when cognitive circuits are engaged; they emphasized that, to be most effective, exercise must be of high intensity, challenging, specific to the motor circuits affected, and engaging to the individual. Exercise as medicine has caught on in the 
public health domain and marketing worlds. But one size does not fit all. Not every person in the exercise studies previously described improved from the same intervention or to the same degree. Why is that? Sound exercise prescription relies on clearly identifying symptoms, understanding the indications for the intervention, and providing the appropriate intensity, frequency, and duration.

In summary, exercise has limited risks and can be a user-friendly, costeffective, and self-motivated moderator of ongoing disease with the potential for restorative and preventative outcomes. Exercise is well accepted in the MS community as a critical component of a healthy lifestyle. The National
Multiple Sclerosis Society (NMSS) recognizes the need for a comprehensive approach when addressing MS symptoms and supports the framework that exercise is a viable treatment in this complex disease. Taking this support one step further, the NMSS convened a Wellness Research Working Group of scientists and patients whose mission is to consolidate evidence supporting a wellness lifestyle, including exercise, with a goal of providing practical recommendations for people living with MS. ${ }^{18}$ Exercise offers a promising path for addressing progressive MS, nervous system repair, wellness, and lifestyle. The scientific community, along with the NMSS and others, must continue to work toward gaining information to establish an evidence-based exercise prescription for people living with MS. $\square$
1. Davis FA, Michael JA, Tomaszewski JS, Fluctuation of motor function in multiple sclerosis related to circadian temperature variations, Dis Nerv Syst, 1973;34:33-6.

2. Petajan $\mathrm{JH}$, Gappmaier E, White AT, et al., Impact of aerobic training on fitness and quality of life in multiple sclerosis, Ann Neurol, 1996;39:432-41.

3. Petajan $\mathrm{JH}$, White AT, Recommendations for physical activity in patients with multiple sclerosis, Sports Med AuckI NZ, 1999;27:179-91.

4. Platta ME, Ensari I, Motl RW, Pilutti LA, Effect of exercise training on fitness in multiple sclerosis: a meta-analysis, Arch Phys Med Rehabil, 2016;97:1564-72.

5. Pilutti LA, Greenlee TA, Motl RW, et al., Effects of exercise training on fatigue in multiple sclerosis: a meta-analysis, Psychosom Med, 2013:75:575-80.

6. Snook EM, Motl RW, Effect of exercise training on walking mobility in multiple sclerosis: a meta-analysis, Neurorehabil Neural Repair, 2009;23:108-16.

7. Latimer-Cheung AE, Pilutti LA, Hicks AL, et al., Effects of exercise training on fitness, mobility, fatigue, and health-related quality of life among adults with multiple sclerosis: a systematic to inform guideline development, Arch Phys Med Rehabil، 2013;94:1800-28.e3.

8. Frohman AN, Okuda DT, Beh S, et al., Aquatic training in MS: neurotherapeutic impact upon quality of life, Ann Clin Trans/ Neurol, 2015:2:864-72.

9. Wiens ME, Reimer MA, Guyn HL, Music therapy as a treatment method for improving respiratory muscle strength in patients with advanced multiple sclerosis: a pilot study, Rehabil Nurs Off J Assoc Rehabil Nurses, 1999;24:74-80.

10. Keller JL, Fritz N, Chiang CC, et al., Adapted resistance training improves strength in eight weeks in individuals with multiple sclerosis, J Vis Exp, 2016;107:e53449. doi:10.3791/53449.

11. Zackowski KM, Smith SA, Reich DS, et al., Sensorimotor dysfunction in multiple sclerosis and column-specific magnetization transfer-imaging abnormalities in the spinal cord Brain, 2009;132:1200-9.

12. Tecchio $F$, Cancelli $A$, Cottone $C$, et al., Brain plasticity effects of neuromodulation against multiple sclerosis fatigue, Front Neurol, 2015;6:1-8.

13. Prakash RS, Snook EM, Erickson KI, et al., Cardiorespiratory fitness: a predictor of cortical plasticity in multiple sclerosis,
Neurolmage, 2007;34:1238-44.

14. Mifflin KA, Frieser E, Benson C, et al., Voluntary wheel running differentially affects disease outcomes in male and female mice with experimental autoimmune encephalomyelitis,

I Neuroimmunol, 2017:305:135-44.

15. Souza PS, Goncalves ED, Pedroso GS, et al., Physical exercise attenuates experimental autoimmune encephalomyelitis by inhibiting peripheral immune response and blood-brain barrier disruption, Mol Neurobiol, 2016. doi:10.1007/s12035-016-0014-0.

16. Benson C, Paylor JW, Tenorio G, et al., Voluntary wheel running delays disease onset and reduces pain hypersensitivity in early experimental autoimmune encephalomyelitis (EAE), Exp Neurol, 2015;271:279-90.

17. Jakowec MW, Wang Z, Holschneider D, et al., Engaging cognitive circuits to promote motor recovery in degenerative disorders. Exercise as a learning modality, J Hum Kinet, 2016;52:35-51.

18. Motl RW, Mowry EM, Ehde DM, et al., Wellness and multiple sclerosis: the National MS Society establishes a Wellness Research Working Group and research priorities, Mult Scler 2017. doi: $10.1177 / 1352458516687404$ 